$$
\begin{aligned}
& a_{1} \frac{\mathrm{d} x^{\prime}}{\mathrm{d} t}+a_{2} \frac{\mathrm{d} y^{\prime}}{\mathrm{d} t}+a_{3} \frac{\mathrm{d} z^{\prime}}{\mathrm{d} t}=\frac{\mathrm{d} x}{\mathrm{~d} t}-r_{1} y+q_{1} z+g_{1} \\
& b_{1} \frac{\mathrm{d} x^{\prime}}{\mathrm{d} t}+b_{2} \frac{\mathrm{d} y^{\prime}}{\mathrm{d} t}+b_{3} \frac{\mathrm{d} z^{\prime}}{\mathrm{d} t}=\frac{\mathrm{d} y}{\mathrm{~d} t}-p_{1} z+r_{1} x+g_{2} \\
& c_{1} \frac{\mathrm{d} x^{\prime}}{\mathrm{d} t}+c_{2} \frac{\mathrm{d} y^{\prime}}{\mathrm{d} t}+c_{3} \frac{\mathrm{d} z^{\prime}}{\mathrm{d} t}=\frac{\mathrm{d} z}{\mathrm{~d} t}-q x+p y+g_{3}
\end{aligned}
$$

und daher die lebendige Kraft der Relativbewegung:

$$
T^{\prime}=\frac{1}{2} \Sigma m_{i}\left[\left(\begin{array}{c}
\mathrm{d} x_{i} \\
\mathrm{~d} t
\end{array}-r_{1} y_{i}+q_{1} z_{i}+g_{1}\right)^{2}+\left(\frac{\mathrm{d} y_{i}}{\mathrm{~d} t}-p_{1} z_{i}+r_{1} x_{i}+g_{2}\right)^{2}+\left(\begin{array}{l}
\mathrm{d} z_{i} \\
\mathrm{~d} t
\end{array}-q_{1} x_{i}+p_{1} y_{i}+g_{3}\right)^{2}\right]
$$

Soll $T^{\prime}$ ein Minimum werden, so muss zunächst sein: und daher auch:

$$
\frac{\partial T^{\prime}}{\partial g_{1}}=\frac{\partial T^{\prime}}{\partial g_{2}}=\frac{\partial T^{\prime}}{\partial g_{3}}=0 \text {, }
$$

also mit Rücksicht auf die Gleichungen (XVI):

$$
\begin{aligned}
& a_{1} \Sigma m_{i} \frac{\mathrm{d} x_{i}^{\prime}}{\mathrm{d} t}+a_{2} \Sigma m_{i} \frac{\mathrm{d} y_{i}^{\prime}}{\mathrm{d} t}+a_{3} \Sigma m_{i} \frac{\mathrm{d} z_{i}^{\prime}}{\mathrm{d} t}=0 \\
& b_{1} \Sigma m_{i} \frac{\mathrm{d} x_{i}^{\prime}}{\mathrm{d} t}+b_{2} \Sigma m_{i} \frac{\mathrm{d} y_{i}^{\prime}}{\mathrm{d} t}+b_{3} \Sigma m_{i} \frac{\mathrm{d} z_{i}^{\prime}}{\mathrm{d} t}=0 \\
& c_{1} \Sigma m_{i} \frac{\mathrm{d} x_{i}^{\prime}}{\mathrm{d} t}+c_{2} \Sigma m_{i} \frac{\mathrm{d} y_{i}^{\prime}}{\mathrm{d} t}+c_{3} \Sigma m_{i} \frac{\mathrm{d} z_{i}^{\prime}}{\mathrm{d} t}=0 \\
& \frac{\partial T^{\prime}}{\partial p_{1}}=\frac{\partial T^{\prime}}{\partial q_{1}}=\frac{\partial T^{\prime}}{\partial r_{1}}=\circ \\
& \Sigma m_{i}\left[-\left(b_{1} \frac{\mathrm{d} x_{i}^{\prime}}{\mathrm{d} t}+b_{2} \frac{\mathrm{d} y_{i}^{\prime}}{\mathrm{d} t}+b_{3} \frac{\mathrm{d} z_{i}^{\prime}}{\mathrm{d} t}\right) z_{i}+\left(c_{1} \frac{\mathrm{d} x_{i}^{\prime}}{\mathrm{d} t}+c_{2} \frac{\mathrm{d} y_{i}^{\prime}}{\mathrm{d} t}+c_{3} \frac{\mathrm{d} z_{i}^{\prime}}{\mathrm{d} t}\right) y_{i}\right]=0
\end{aligned}
$$$$
\Sigma m_{i} \frac{\mathrm{d} x_{i}^{\prime}}{\mathrm{d} t}=\Sigma m_{i} \frac{\mathrm{d} y_{i}^{\prime}}{\mathrm{d} t}=\Sigma m_{i} \frac{\mathrm{d} \boldsymbol{z}_{i}^{\prime}}{\mathrm{d} t}=0
$$

d.h. bei der Relativbewegung mit dem Minimum von leben-

etc. oder bei Benutzung der Gleichungen (V) und (XVII) und der bekannten Beziehungen der orthogonalen Substitution $b_{2} c_{3}-c_{2} b_{3}=a_{1}$ etc.:

$$
a_{1} \Sigma m_{i}\left(y_{i}^{\prime} \frac{\mathrm{d} z_{i}^{\prime}}{\mathrm{d} t}-z_{i}^{\prime} \frac{\mathrm{d} y_{i}^{\prime}}{\mathrm{d} t}\right)+a_{2} \Sigma m_{i}\left(z_{i}^{\prime} \frac{\mathrm{d} x_{i}^{\prime}}{\mathrm{d} t}-x_{i}^{\prime} \frac{\mathrm{d} z_{i}^{\prime}}{\mathrm{d} t}\right)+a_{3} \Sigma m_{i}\left(x_{i}^{\prime}{ }^{\prime} y_{i}^{\prime}-y_{i}^{\prime} \frac{\mathrm{d} x_{i}^{\prime}}{\mathrm{d} t}\right)=。
$$

und die beiden aus dieser durch Vertauschung der $a$ mit $b$ und $c$ hervorgehenden. Daher also auch zuletzt:

$$
\Sigma m_{i}\left(y_{i}^{\prime} \frac{\mathrm{d} z_{i}^{\prime}}{\mathrm{d} t}-z_{i}^{\prime} \frac{\mathrm{d} y_{i}^{\prime}}{\mathrm{d} t}\right)=\Sigma m_{i}\left(z_{i}^{\prime} \frac{\mathrm{d} x_{i}^{\prime}}{\mathrm{d} t}-\dot{x}_{i}^{\prime} \frac{\mathrm{d} z_{i}^{\prime}}{\mathrm{d} t}\right)=\Sigma m_{i}\left(x_{i}^{\prime} \frac{\mathrm{d} y_{i}^{\prime}}{\mathrm{d} t}-y_{i}^{\prime} \frac{\mathrm{d} x_{i}^{\prime}}{\mathrm{d} t}\right)=。
$$

d.h. für die Relativbewegung mit der geringsten lebendigen $\mathrm{K}$ raft sind die Flächenintegrale $=0$. Bekanntlich sind die Flächenintegrale bei der absoluten Bewegung des Systems constant und sie bleiben auch constant, wenn dabei von der Bewegung des Schwerpunktes abgesehen wird. Diese drei Constanten bestimmen die von Laplace eingeführte sun. veränderliche Ebenes. Unsere Relativbewegung ist im all-

Charlottenburg, r898 Mai 21. gemeinen Falle von dieser absoluten Bewegung durchaus verschieden und nur wenn die drei Flächenintegrale verschwinden, die unveränderliche Ebene daher illusorisch wird, ist die absolute Bewegung zugleich auch die mit der geringsten lebendigen Kraft ausgestattete unter allen Relativbewegungen.

\title{
Auszug aus einem Schreiben an den Herausgeber
}

\section{betr. das Interpolationsverfahren von Tschebyschef.}

Einer freundlichen Mittheilung von Herrn Prof. Lüroth verdanke ich die Notiz, dass die Reihenumkehrung, die ich in Nr. 349 I der A. N. zur Herleitung des Tschebyschef'schen Interpolationsverfahrens benutzt habe, sich bereits in allgemeinerer Gestalt bei Möbius findet, und zwar in dem Auf- satze ,Ueber eine besondere Art von Umkehrung der Reihen «, (Crelle's Journal Band X, Seite 105 und Gesammelte Werke, Band IV, Seite 588). Setzt man den Satz von Möbius als bekannt voraus, so erkennt man, dass die Tschebyschef'sche Reihenentwickelung sich mit wenigen Zeilen erledigen lässt.

Leipzig, 1898 Aug. I 3 .

H. Bruns. 